\title{
Quantifying ERK activity in response to inhibition of the BRAFV600E-MEK-ERK cascade using mathematical modelling
}

\author{
Sara J. Hamis $\mathbb{D D}^{1 凶}$, Yury Kapelyukh ${ }^{2}$, Aileen McLaren ${ }^{2}$, Colin J. Henderson ${ }^{2}$, C. Roland Wolf ${ }^{2}$ and Mark A. J. Chaplain ${ }^{1}$
}

(c) The Author(s) 2021

BACKGROUND: Simultaneous inhibition of multiple components of the BRAF-MEK-ERK cascade (vertical inhibition) has become a standard of care for treating BRAF-mutant melanoma. However, the molecular mechanism of how vertical inhibition synergistically suppresses intracellular ERK activity, and consequently cell proliferation, are yet to be fully elucidated.

METHODS: We develop a mechanistic mathematical model that describes how the mutant BRAF inhibitor, dabrafenib, and the MEK inhibitor, trametinib, affect BRAFV600E-MEK-ERK signalling. The model is based on a system of chemical reactions that describes cascade signalling dynamics. Using mass action kinetics, the chemical reactions are re-expressed as ordinary differential equations that are parameterised by in vitro data and solved numerically to obtain the temporal evolution of cascade component concentrations.

RESULTS: The model provides a quantitative method to compute how dabrafenib and trametinib can be used in combination to synergistically inhibit ERK activity in BRAFV600E-mutant melanoma cells. The model elucidates molecular mechanisms of vertical inhibition of the BRAFV600E-MEK-ERK cascade and delineates how elevated BRAF concentrations generate drug resistance to dabrafenib and trametinib. The computational simulations further suggest that elevated ATP levels could be a factor in drug resistance to dabrafenib.

CONCLUSIONS: The model can be used to systematically motivate which dabrafenib-trametinib dose combinations, for treating BRAFV600E-mutated melanoma, warrant experimental investigation.

British Journal of Cancer (2021) 125:1552-1560; https://doi.org/10.1038/s41416-021-01565-w

\section{BACKGROUND}

Mitogen-activated protein kinase (MAPK) pathways are present in all eukaryotic cells and play a pivotal role in cellular processes linked to proliferation, differentiation and apoptosis [1]. A general MAPK pathway consists of a three-tiered cascade, with a MAPK kinase kinase (MAPKKK) on the top tier, a MAPK kinase (MAPKK) on the middle tier, and a MAPK on the bottom tier. Via sequential kinase activation, signals can propagate through the MAPK cascade in which the activated MAPKKK phosphorylates, and thereby activates the MAPKK, which in turn phosphorylates, and thereby activates the MAPK.

Several MAPKKK-MAPKK-MAPK cascades are present in eukaryotic cells, but in this study, we focus our attention on one such cascade: the BRAF-MEK-ERK cascade. Mutations in this signalling pathway have been identified as drivers for tumour development and growth, and therefore this cascade is of particular interest in the treatment of cancer. Upon activation, ERK can phosphorylate numerous substrates in the cytoplasm and the nucleus, and thereby regulate gene expression directly, by phosphorylating transcription factors such as Elk, Ets and Myc, and indirectly, by acting on substrates that in turn can modify transcription factors [2]. These phosphorylation events, which are controlled by both the amplitude and the duration of ERK activation, are crucial in regulating intracellular processes, including cell proliferation.

In the early 2000s, it was established that BRAF oncogene mutations occur in a majority of melanomas [3]. In melanomas harbouring the BRAFV600E mutation, the most common BRAF mutation [4], the BRAF kinase, is constitutively activated and thus signalling through the BRAF-MEK-ERK cascade is hyperactivated and always turned on, which can result in uncontrolled cell proliferation $[5,6]$. As a consequence, small-molecule inhibitors that target the BRAF-MEK-ERK cascade have been developed, with these inhibitors acting to inhibit the enzymatic activity of the kinases in the cascade [7]. Such inhibitors ultimately suppress the amount of activated ERK within a cell and, by extension, the cell's proliferative abilities. Such drugs have been shown to have profound anti-tumour effects.

Vertical inhibition describes a treatment approach in which two or more tiers in a cascade, such as the BRAF-MEK-ERK cascade, are concurrently targeted [8]. In this paper, we specifically investigate drug responses to monotherapies and combination therapies that include the BRAF inhibitor dabrafenib (DBF) and the MEK inhibitor trametinib (TMT). DBF is adenosine triphosphate (ATP)-competitive inhibitor with specificity for the MAPKKK BRAFV600E, and TMT

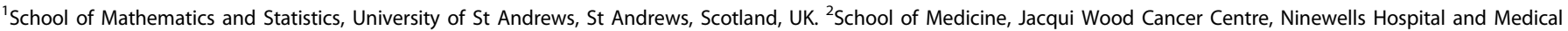

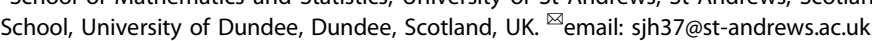

Received: 13 May 2021 Revised: 6 September 2021 Accepted: 21 September 2021

Published online: 7 October 2021 


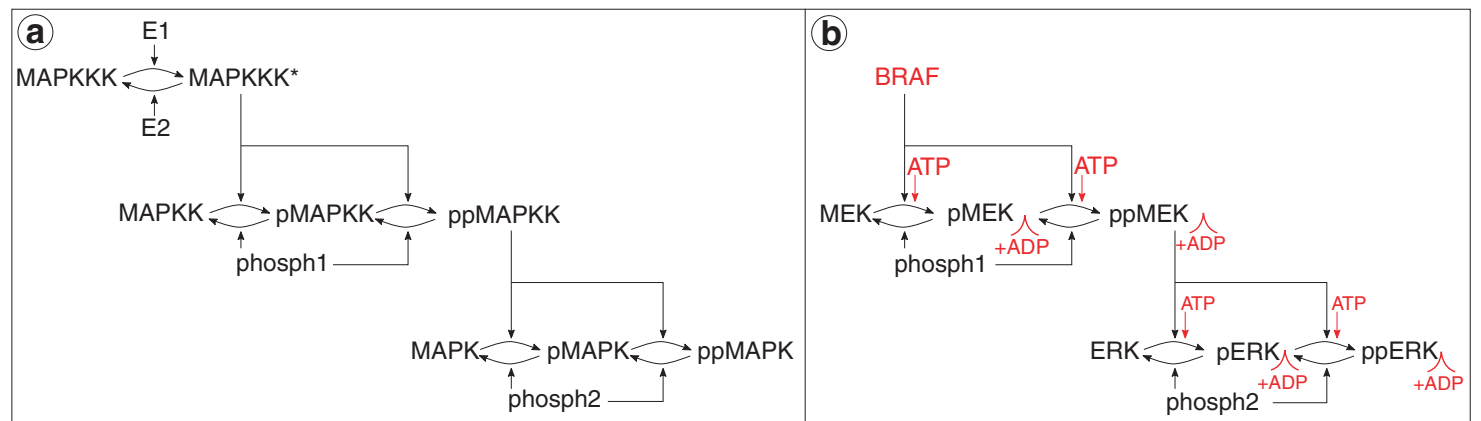

Fig. 1 Cascade models. a The three-tiered general MAPKKK-MAPKK-MAPK cascade of Huang and Ferrell [21]. The star $(*)$ denotes activated MAPKKK, and E1 and E2 are unspecified enzymes that, respectively, activate and inactivate MAPKKK. The prefixes $p$ and pp denote singly and doubly phosphorylated proteins, and phosph1 and phosph2 are phosphatases that dephosphorylate MAPKK and MAPK, respectively. b Our modified BRAF-MEK-ERK cascade in BRAFV600E-mutant melanoma, in which a specific MAPKKK (BRAF), MAPKK (MEK) and MAPK (ERK) are considered. BRAF is constitutively activated, and therefore the top cascade tier is reduced to a single node, activated BRAF. The modified cascade incorporates ATP-dependent substrate phosphorylation at the sites shown. Differences in the cascade structure between Huang and Ferrell's original model, and our model, are marked in red in (b).

selectively inhibits the MAPKKs MEK1 and MEK2 [9]. TMT is an allosteric inhibitor as it inhibits the enzymatic activity of MEK1 and MEK2 by binding to a site that is distinct from the ATP-binding site [10]. In 2011, the first selective inhibitor of mutated BRAF, vemurafenib, was approved by the US Food and Drug Administration for treating BRAF-mutant metastatic or unresectable melanoma [11]. Thereafter, selective BRAF inhibitors replaced the previously used chemotherapies in treating BRAFV600E and BRAFV600K mutant melanoma in clinics, which significantly improved patient survival [12]. However, drug resistance to BRAF-inhibiting monotherapies commonly develops within 6-8 months of treatment [13-15].

In current treatment protocols, combined BRAF and MEK inhibition is a standard of care for treating BRAFV600E-mutant melanoma $[13,14,16,17]$. Compared to BRAF-inhibiting monotherapies, such combination therapies have been shown to delay the onset of drug resistance; in a clinical phase 3 trial, the median progression-free survival was respectively reported to be 5.8 and 9.4 months for DBF mono treatments and DBF-TMT combination treatments [14]. This, yet rapid, onset of drug resistance suggests that current treatment regimens create a pressure that selects for drug-resistant tumour subclones [15].

Multiple and diverse mechanisms have been empirically identified as drivers for resistance to BRAF inhibitors, and one important such mechanism is BRAFV600E amplification [18]. In a multicenter meta-analysis study of clinically observed resistance mechanisms in BRAFV600E-mutated melanoma, quantitative genomic DNA PCR testing showed that BRAFV600E/K amplification was present in 120 out of 132 patient biopsies [19]. Xue et al. moreover experimentally showed that increased BRAFV600E concentrations led to a growth advantage in A375 melanoma cells in vitro, when the cells were treated with RAF, MEK or ERK inhibitors [4]. Interestingly, in the absence of these drugs, BRAFV600E amplification did not yield a growth advantage and thus the authors concluded that the magnitude of BRAFV600E amplification required for sustained BRAFV600E-MEK-ERK signalling is drug-dependent. Xue et al. referred to this magnitude as the fitness threshold. As part of their study, the authors further showed, using patient-derived xenografts, that an increased fitness threshold can be obtained via intermittent administration of vertical MAPK inhibition.

Xue et al.'s fitness threshold model can be used to link drug responses with the evolutionary selection of subclones within a tumour, but does not explain why; on an intracellular level, an increase in BRAFV600E concentration leads to a growth advantage only in the presence of drugs. However, a recent study by Sale et al. demonstrated that BRAFV600E-amplified cells can become addicted to MEK inhibitors and that ERK1 or ERK2 activation post drug withdrawal drives $\mathrm{G} 1$ cell-cycle arrest, senescence and cell death [20]. This indicates that, during drug withdrawal, BRAFV600E-amplified cells have a proliferative disadvantage compared to non-BRAFV600E-amplified cells.

In order to further shed light on the effects of vertical inhibition of the BRAFV600E-MEK-ERK cascade at a molecular level, we here develop a mathematical model that mechanistically captures cascade dynamics in the presence of DBF and TMT. Huang and Ferrell developed the first mathematical model of a general MAPK pathway [21], consisting of a three-tiered cascade in which double phosphorylation of the MAPKKK, MAPKK and MAPK substrates is required for activation. Using mass action kinetics, Huang and Ferrell formulated the MAPK cascade in terms of a system of ordinary differential equations (ODEs), which they solved numerically to simulate signalling dynamics within the cascade. Notably, the authors showed that the structure of the MAPK cascade yields ultra-sensitive signalling dynamics. In this study, we modify Huang and Ferrell's model to capture cellular signalling dynamics in a specific MAPK pathway: the BRAF-MEK-ERK cascade in BRAFV600Emutant melanoma. As an extension to Huang and Ferrell's model, we introduce ATP-dependent substrate phosphorylation and molecular drug actions of DBF and TMT.

\section{METHODS}

We develop a mathematical model that simulates the temporal BRAF-MEKERK signalling dynamics in BRAFV600E-mutant melanoma. In order to do this, we first map out the structure of the BRAF-MEK-ERK cascade and identify the drug actions of DBF and TMT that, respectively, inhibit enzymatic BRAF and MEK activity. From the cascade structure and drug actions, we formulate a system of chemical reactions that captures intracellular signalling dynamics. Using mass action kinetics, the system of chemical reactions is thereafter re-formulated as a system of ODEs, which are solved numerically in order to simulate the temporal evolution of signalling molecule concentrations. A model output of particular importance is the concentration of activated ERK over time, as the ultimate goal of vertical inhibition of the BRAFV600E-MEK-ERK pathway is to suppress cells' activated ERK levels and, by extension, their proliferative abilities. All model parameters (i.e. kinetic constants and total intracellular protein concentrations) are obtained from data available in the literature [21-29].

\section{The BRAF-MEK-ERK cascade}

We modify Huang and Ferrel's cascade model [21] to specifically capture the signalling dynamics of the BRAF-MEK-ERK cascade in BRAFV600Emutant melanoma. Both Huang and Ferrell's general MAPKKK-MAPKK- 


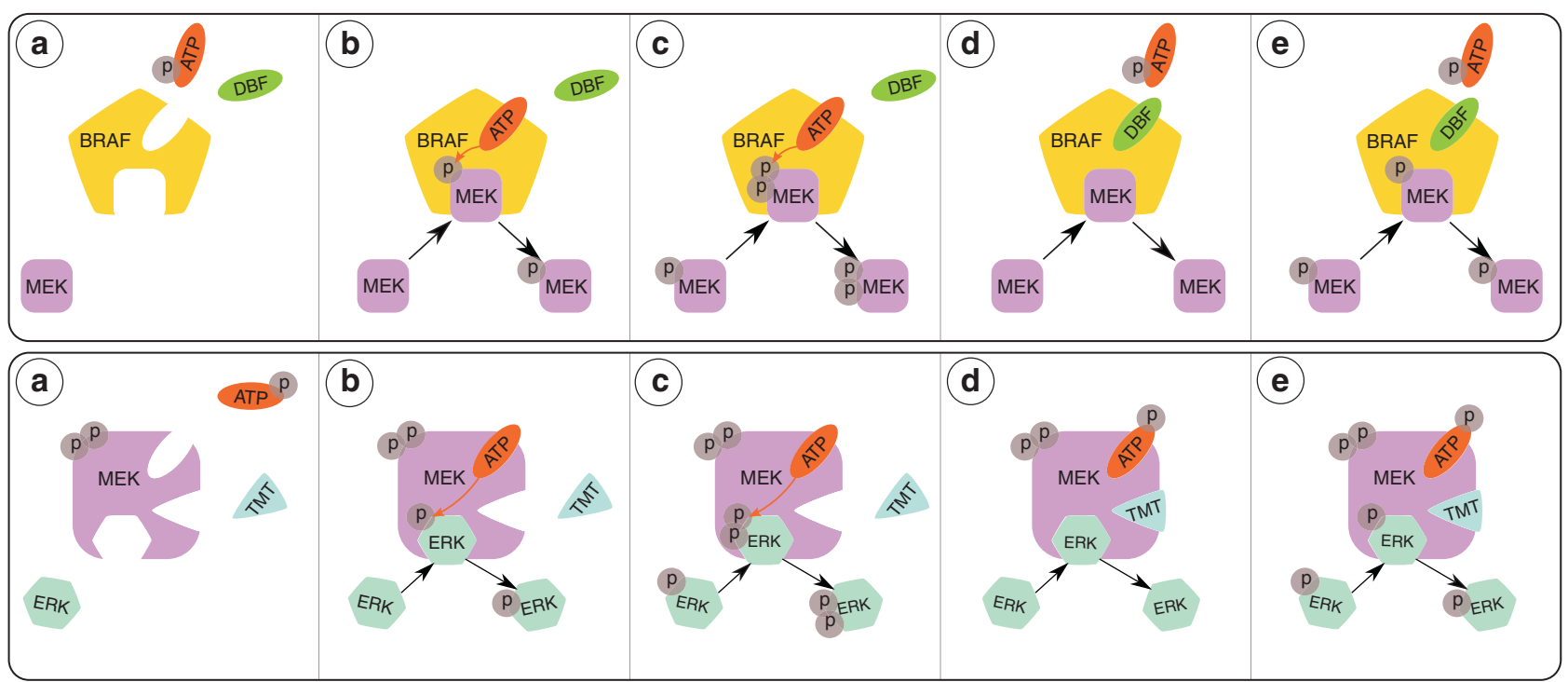

Fig. 2 Schematics of the mathematical models that describe dabrafenib (DBF) and trametinib (TMT) drug actions. (Top) a We consider two BRAF binding sites: one that can bind MEK and one that can bind ATP or dabrafenib. BRAF-bound ATP is necessary for the phosphorylation of MEK. $\mathbf{b}$ If ATP and MEK are bound to BRAF, then MEK is phosphorylated to the singly phosphorylated form pMEK. $\mathbf{c}$ If ATP and pMEK are bound to BRAF, then pMEK is phosphorylated to the doubly phosphorylated form ppMEK. d, e BRAF-bound dabrafenib prevents ATP from binding to BRAF, and thereby inhibits the phosphorylation of the substrates MEK and PMEK. (Bottom) a We consider three MEK-binding sites: one that can bind ERK, one that can bind ATP and one that can bind trametinib. ppMEK-bound ATP is necessary for the phosphorylation of ERK. $\mathbf{b}$ If ERK and ATP, but not TMT, are bound to pPMEK, then ERK is phosphorylated to the singly phosphorylated form pERK. c If pERK and ATP, but not TMT, are bound to ppMEK, then pERK is phosphorylated to the doubly phosphorylated form ppERK. d, e If TMT is bound to ppMEK, the phosphorylation of the substrates ERK and PERK is inhibited.

MAPK cascades and our problem-specific cascade are, respectively, illustrated in Fig. 1a, b. Here, and throughout this work, singly and doubly phosphorylated proteins are, respectively, denoted by the prefixes $\mathrm{p}$ and $\mathrm{pp}$. Note that double phosphorylation is required for substrate activation and that phosphatases (phosphs) dephosphorylate singly and doubly phosphorylated MAPKK/MEK and MAPK/ERK (Fig. 1). In our model, BRAF phosphorylates MEK and once MEK has been doubly phosphorylated, it in turn phosphorylates ERK, which becomes activated when doubly phosphorylated. As BRAF is constitutively activated in BRAFV600E-mutant melanoma [6], we have reduced the top tier in our modified cascade to contain only activated BRAF (Fig. 1b). Since the model developed in this study will include the drug actions of DBF, which is an ATP-competitive inhibitor, and TMT, which is an allosteric ATP inhibitor, we extend Huang and Ferrell's model to include ATP-dependent substrate phosphorylation. Thus, in our model, when a substrate and an ATP molecule bind to an enzyme (in the absence of drugs), the ATP molecule donates a phosphate group to the substrate, and the ATP converts to adenosine diphosphate (ADP) after the metabolic process (Fig. 1b).

\section{BRAF and MEK inhibitors}

We model the pharmacodynamic actions of the two drugs DBF and TMT. DBF is a potent and selective BRAF inhibitor, which is ATP-competitive [30]. As illustrated schematically in Fig. 2 (top), DBF can thus bind to the ATP site of a BRAF molecule, and thereby inhibit BRAF-ATP reactions and the downstream phosphorylation of MEK and PMEK. TMT is classified as an allosteric MEK $1 / 2$ inhibitor, as it binds to a binding site that is distinct from, but adjacent to, the ATP-binding site on the MEK molecule [31]. This is illustrated schematically in Fig. 2 (bottom). MEK-bound TMT prevents MEK from phosphorylating the substrates ERK and pERK.

\section{The system of reactions}

By combining the cascade illustrated in Fig. $1 \mathrm{~b}$, with the drug actions that are schematically summarised in Fig. 2, we formulate a system of chemical reactions that describes the signalling dynamics in the BRAFV600E-MEKERK cascade subjected to drugs. This results in a system of 36 chemical reactions (R.1-R.36) that are outlined in Fig. 3, where the order of the binding events is considered.

\section{The system of ordinary differential equations}

We use the law of mass action to re-express the system of reactions (R.1-R.36) as a system of ODEs. In the system of ODEs, the dependent variables are the concentrations of the signalling molecules that occur in (R.1-R.36), and the independent variable is time. In short, the law of mass action says that the rate of a chemical reaction is proportional to the product of the masses of the reactants. Thus, the first reaction, R.1, which includes the molecules BRAF, ATP and BRAF.ATP, yields the following contributions to the system of ODEs:

$$
\begin{aligned}
& \frac{\mathrm{d}[\mathrm{BRAF}]}{\mathrm{d} t}=-a_{1}[\mathrm{BRAF}][\mathrm{ATP}]+d_{1}[\mathrm{BRAF} \cdot \mathrm{ATP}], \\
& \frac{\mathrm{d}[\mathrm{ATP}]}{\mathrm{d} t}=-a_{1}[\mathrm{BRAF}][\mathrm{ATP}]+d_{1}[\mathrm{BRAF} \cdot \mathrm{ATP}], \\
& \frac{\mathrm{d}[\mathrm{BRAF} \cdot \mathrm{ATP}]}{\mathrm{d} t}=+a_{1}[\mathrm{BRAF}][\mathrm{ATP}]-d_{1}[\mathrm{BRAF} \cdot \mathrm{ATP}],
\end{aligned}
$$

where concentrations are denoted by square brackets surrounding the signalling molecule of interest, and $t$ denotes time. Note that the righthand sides of the above equations are subsequently extended to include more equations derived from other reactions in the system of reactions outlined in Fig. 3. The full system of ODEs $(0.1-0.36)$ is available in the Supplementary Material (SM1). In this work, we combine (0.1-0.36) with a set of system conservation laws (C.1-C.7 in SM1) to formulate a system of differential-algebraic equations that we solve numerically to obtain the temporal evolution of the system molecule concentrations. The conservation laws ensure that the total concentrations of the kinases BRAF, MEK and ERK (in free, bound, non-phosphorylated, singly phosphorylated and doubly phosphorylated form), phosphatases (in free and bound form) and drugs (in free and bound form) are conserved within the system. 


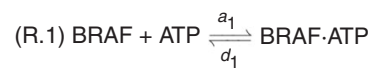

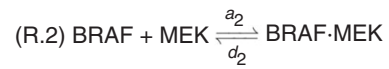

(R.3) BRAF + pMEK $\frac{a_{2}}{\underset{d_{2}}{\rightleftharpoons}}$ BRAF.pMEK

(R.4) BRAF.ATP + MEK $\frac{a_{2}}{\underset{d_{2}}{\rightleftharpoons}}$ BRAF.ATP.MEK $\stackrel{k_{12}}{\longrightarrow}$ BRAF + ADP + pMEK

(R.5) BRAF.ATP + pMEK $\frac{a_{2}}{d_{2}}$ BRAF.ATP.pMEK $\stackrel{k_{12}}{\longrightarrow}$ BRAF + ADP + ppMEK

(R.6) BRAF.MEK + ATP $\frac{a_{1}}{d_{1}}$ BRAF.ATP.MEK $\stackrel{k_{12}}{\longrightarrow}$ BRAF + ADP + pMEK

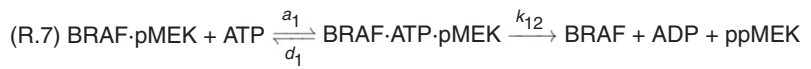

(R.8) pMEK + phosph1 $\underset{d_{3}}{\stackrel{a_{3}}{\rightleftharpoons}}$ pMEK·phosph1 $\stackrel{k_{3}}{\longrightarrow}$ MEK + phosph1

(R.9) ppMEK + phosph1 $\underset{d_{3}}{\stackrel{a_{3}}{\rightleftharpoons}}$ ppMEK.phosph1 $\stackrel{k_{3}}{\longrightarrow}$ pMEK + phosph1

$(\mathrm{R} .10)^{*} \mathrm{BRAF}+\mathrm{DBF} \underset{d_{4}}{\stackrel{a_{4}}{\rightleftharpoons}}$ BRAF.DBF

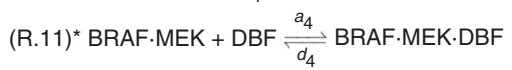

$(R .12)^{*}$ BRAF.pMEK + DBF $\frac{a_{4}}{\underset{d_{4}}{\rightleftharpoons}}$ BRAF.pMEK.DBF

$(\mathrm{R} .13)^{*}$ BRAF.DBF + MEK $\frac{a_{2}}{\frac{d_{2}}{d_{2}}}$ BRAF.MEK.DBF

$(\mathrm{R} .14)^{\star}$ BRAF.DBF + pMEK $\frac{a_{2}}{\frac{d_{2}}{\longrightarrow}}$ BRAF.pMEK.DBF

(R.15) ppMEK + ATP $\underset{d_{5}}{\stackrel{a_{5}}{\rightleftharpoons}}$ ppMEK·ATP

(R.16) ppMEK + ERK $\underset{d_{6}}{\stackrel{a_{6}}{\rightleftharpoons}}$ ppMEK.ERK

(R.17) ppMEK + pERK $\underset{d_{6}}{\stackrel{a_{6}}{\rightleftharpoons}}$ ppMEK.pERK

(R.18) ppMEK $\cdot$ ATP + ERK $\underset{d_{6}}{\stackrel{a_{6}}{\rightleftharpoons}}$ ppMEK $\cdot$ ATP $\cdot$ ERK $\stackrel{k_{56}}{\longrightarrow}$ ppMEK + ADP + pERK
(R.19) ppMEK·ATP + pERK $\underset{\frac{a_{6}}{d_{6}}}{\rightleftharpoons}$ ppMEK·ATP.pERK $\stackrel{k_{56}}{\longrightarrow}$ ppMEK + ADP + ppERK

(R.20) ppMEK $\cdot$ ERK + ATP $\frac{a_{5}}{d_{5}}$ ppMEK $\cdot$ ATP $\cdot$ ERK $\stackrel{k_{56}}{\longrightarrow}$ ppMEK + ADP + ppERK

(R.21) ppMEK.pERK + ATP $\frac{a_{5}}{d_{5}}$ ppMEK ATP.pERK $\stackrel{k_{56}}{\longrightarrow}$ ppMEK + ADP + ppERK

(R.22) pERK + phosph2 $\frac{a_{7}}{d_{7}}$ pERK.phosph2 $\stackrel{k_{7}}{\longrightarrow}$ ERK + phosph2

(R.23) ppERK + phosph2 $\frac{a_{7}}{\underset{d_{7}}{\rightleftharpoons}}$ ppERK $\cdot$ phosph $2 \stackrel{k_{7}}{\longrightarrow}$ pERK + phosph2

$(\text { R.24) })^{\star \star}$ ppMEK + TMT $\underset{d_{8}}{\stackrel{a_{8}}{\rightleftharpoons}}$ ppMEK $\cdot$ TMT

$\left(\right.$ R.25) ${ }^{\star *}$ ppMEK·ATP + TMT $\underset{d_{8}}{\stackrel{a_{8}}{\rightleftharpoons}}$ ppMEK·ATP.TMT

$\left(\right.$ R.26) ${ }^{* *}$ ppMEK $\cdot$ ERK + TMT $\underset{d_{8}}{\stackrel{a_{8}}{\rightleftharpoons}}$ ppMEK·ERK·TMT

$(\text { R.27) })^{\star *}$ ppMEK.pERK + TMT $\frac{a_{8}}{\frac{d_{8}}{\rightleftharpoons}}$ ppMEK.pERK·TMT

$(\text { R.28) })^{\star \star}$ ppMEK·ERK.ATP + TMT $\frac{a_{8}}{\underset{d_{8}}{\rightleftharpoons}}$ ppMEK·ERK.ATP.TMT

$\left(\right.$ R.29) ${ }^{\star \star}$ ppMEK.pERK.ATP + TMT $\frac{a_{8}}{\underset{d_{8}}{\rightleftharpoons}}$ ppMEK.pERK.ATP.TMT

$(\text { R.30 })^{\star \star}$ ppMEK.TMT + ATP $\underset{d_{5}}{\stackrel{a_{5}}{\rightleftharpoons}}$ ppMEK ATP.TMT

$(\text { R.31 })^{\star *}$ ppMEK·TMT + ERK $\underset{\frac{a_{6}}{d_{6}}}{\rightleftharpoons}$ ppMEK·ERK.TMT

$(\text { R.32) })^{\star \star}$ ppMEK·TMT + pERK $\frac{a_{6}}{d_{6}}$ ppMEK.pERK.TMT

$\left(\right.$ R.33) ${ }^{\star \star}$ ppMEK·TMT.ERK + ATP $\frac{a_{5}}{d_{5}}$ ppMEK·ERK.ATP.TMT

$(\text { R.34 })^{\star *}$ ppMEK·TMT.pERK + ATP $\underset{d_{5}}{\stackrel{a_{5}}{\rightleftharpoons}}$ ppMEK.pERK·ATP.TMT

$\left(\right.$ R.35) ${ }^{\star \star}$ ppMEK·TMT.ATP + ERK $\underset{d_{6}}{\stackrel{a_{6}}{\rightleftharpoons}}$ ppMEK·ERK.ATP.TMT

$\left(\right.$ R.36) ${ }^{\star *}$ ppMEK·TMT.ATP + pERK $\frac{a_{6}}{\underset{d_{6}}{\rightleftharpoons}}$ ppMEK.pERK.ATP.TMT

Fig. 3 System of reactions (R.1-R.36) describing the signalling dynamics in the BRAFV600E-MEK-ERK cascade subjected to the drugs dabrafenib (DBF) and trametinib (TMT). The dot notation (.) denotes complexes of two or more molecules. Reactions involving dabrafenib

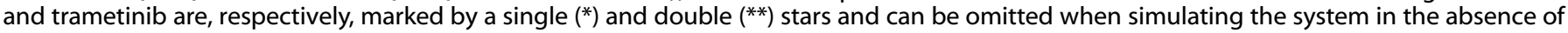
these inhibitors.

\section{Model implementation}

We implement and solve the system of ODEs (0.1-0.36), with conservation laws (C.1-C.7) in silico, in order to simulate the signalling dynamics in the BRAF-MEK-ERK cascade. The in silico model, which is developed using the programing language and computing environment MATLAB, is available on the code hosting platform GitHub. Instructions on how to access, run and modify the code are available in the Supplementary Material (SM2, Fig. SF1).

\section{Model parameters}

The system of reactions (R.1-R.36) includes eight forward rate constants $a_{1}$, $a_{2}, \ldots, a_{8}$, eight reverse rate constants $d_{1}, d_{2}, \ldots, d_{8}$, and four catalytic rate constants $k_{1,2}, k_{3}, k_{5,6}, k_{7}$. The values of these constants are listed in Table ST1 in the Supplementary Material (SM3). Huang and Ferrell [21] argued that, when computing steady-state enzyme levels, it is not the individual rate constants, but rather the Michaelis constants, $K_{\mathrm{mi}}=\left(d_{i}+k_{i}\right) / a_{i}$, that are important. We, therefore, set all forward rate constants $a_{i}$ to be the same so that $a_{j}=a_{1}$ for all $j=2,3, \ldots, 8$. We then use data available in the literature to set the parameter values for $a_{1}$, the eight reverse rate constants and the four catalytic rate constants, as is outlined in the Supplementary Material (SM4). The model initial condition, i.e. a vector that includes the molecule concentrations at the start of the simulation, is also obtained from data in the literature [21, 28]. Initial cascade component concentrations are listed in Table ST2 in the Supplementary Material (SM3).

\section{Results}

Using the in silico model that we have developed, the temporal evolution of the concentration of all signalling components appearing in the system of reactions (R.1-R.36) are computed. In this study, our main model output of interest is activated ERK. For ease of presentation, we define a measure activated ERK, as a time-dependent fraction,

activated ERK $=\frac{[\mathrm{ppERK}](t)}{\mathrm{ERK}_{\mathrm{tot}}}$,

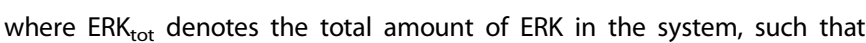
$E R K_{\text {tot }}=[E R K](0)$, as listed in Table ST2 in the Supplementary Material (SM3).

\section{Monotherapy results}

We initially investigate how activated ERK levels within a cell change in response to DBF or TMT monotherapy. The temporal evolution of activated ERK in response to these monotherapies are shown in Fig. $4 a$ and $b$, respectively. Activated ERK levels at specific time points are plotted over intracellular concentrations of BRAF (left panel) and ATP (right panel) for different doses of DBF (Fig. 4c) and TMT (Fig. 4d).

The simulation data show that the modelled DBF treatment responses are highly dynamic in time, and are dependent on both intracellular BRAF and ATP concentrations (Fig. 4a, c). These data also suggest that increased concentrations of BRAF and ATP make the cells less sensitive to DBF. Further, these data show that the activated ERK levels eventually reach the same steady-state value for several DBF-BRAF-ATP concentration combinations (see also Supplementary Material SM5, Fig. SF2). In order for a cell to progress from the $\mathrm{G} 1$ to the $S$ phase of the cell cycle, sustained ERK activity throughout the G1 phase is required, where ERK downregulates several anti-proliferative genes until the S-phase entry [32, 33]. This suggests that, if ERK activity can be suppressed for the duration of the G1 cell-cycle phase, further cell-cycle progression, and thus cell proliferation, can be inhibited.

In response to different TMT doses, activated ERK levels reach distinct steady-state levels (Fig. 4b). Moreover, TMT treatment responses at time 

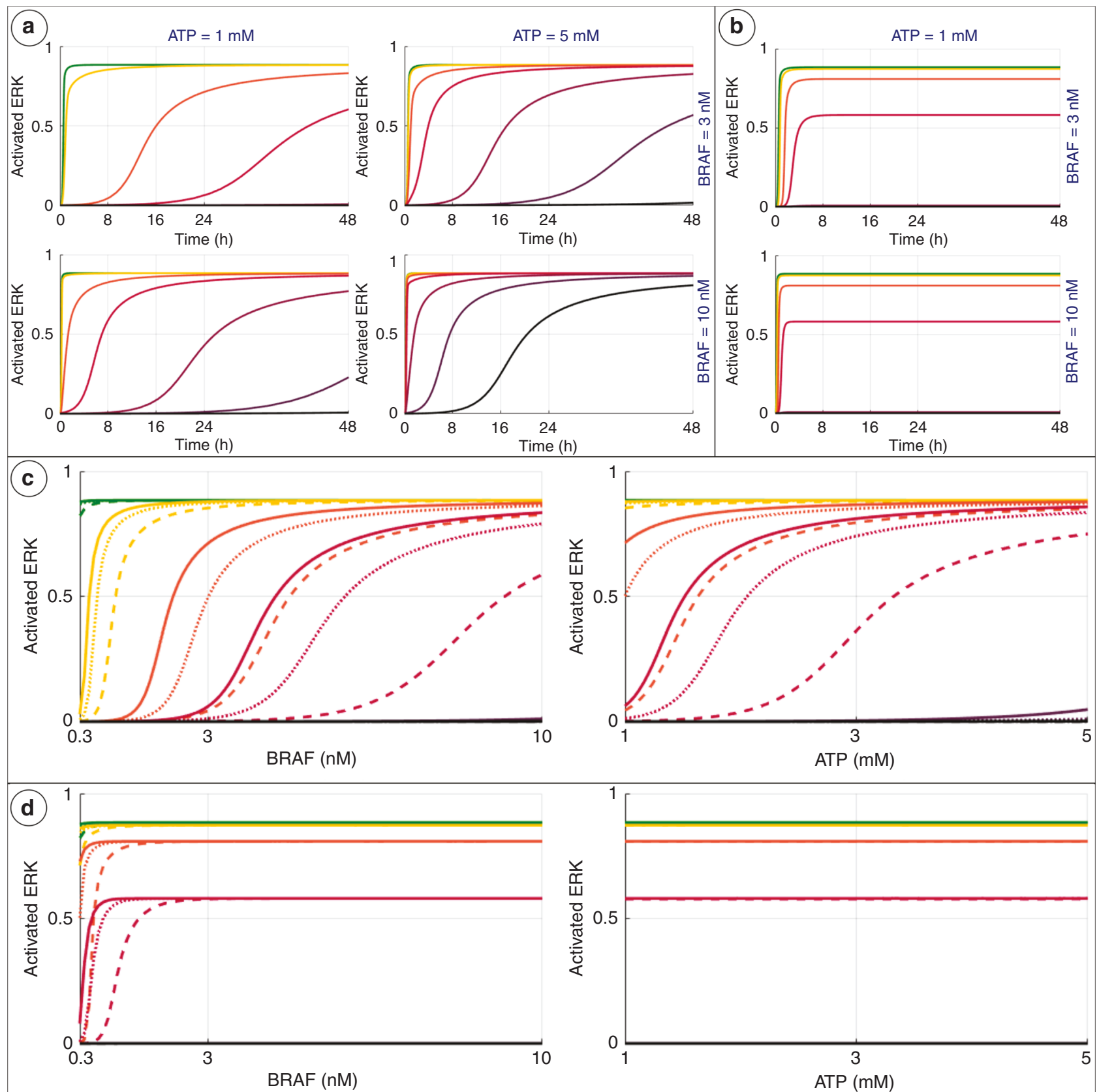

Figure legend

\begin{tabular}{|c|c|c|c|c|c|}
\hline Dabrafenib doses in a \& $\mathbf{c}$ : & $-0 \mu \mathrm{M}-0.1 \mu \mathrm{M}$ & $-0.5 \mu \mathrm{M}$ & $-1 \mu \mathrm{M}$ & $-2.5 \mu \mathrm{M}$ & $-5 \mu \mathrm{M}-10 \mu \mathrm{M}$ \\
\hline Trametinib doses in $\mathbf{b}$ \& $\mathbf{d}$ : & $-0 \mu \mathrm{M}-0.1 \mu \mathrm{M}$ & $-0.5 \mu \mathrm{M}$ & $-1 \mu \mathrm{M}$ & $-2.5 \mu \mathrm{M}$ & $-5 \mu \mathrm{M}-10 \mu \mathrm{M}$ \\
\hline
\end{tabular}

Time points in $\mathbf{c} \& \mathbf{d}$ :

$--8 \mathrm{~h}$

$-12 \mathrm{~h}$

Fig. 4 Plots showing activated ERK levels (as defined in Eq. 2) in response to monotherapies of dabrafenib or trametinib, where the drug doses are indicated in the legend at the bottom of the figure. a The temporal evolution of activated ERK. Results are shown for different intracellular concentrations of BRAF, ATP and dabrafenib (DBF). b The temporal evolution of activated ERK. Results are shown for different intracellular concentrations of BRAF and trametinib (TMT). c, d Activated ERK levels are plotted over intercellular BRAF concentrations, where ATP concentrations are fixed at the baseline value $1 \mathrm{mM}$ (left). Activated ERK levels plotted over intercellular ATP concentrations, where BRAF concentrations are fixed at the baseline value $3 \mathrm{nM}$ (right). Results at 8,16 and $24 \mathrm{~h}$ are shown in dashed, dotted and solid lines, respectively, for different doses of dabrafenib (c) and trametinib (d).

points after $8 \mathrm{~h}$ are only sensitive to BRAF variations for low BRAF concentrations (under $3 \mathrm{nM}$ ) and the efficacy of TMT treatments is not sensitive to variations in ATP within the range of concentrations tested (Fig. 4d).

\section{Combination therapy results}

We next evaluate the effect of combination therapies involving DBF and TMT on treatment outcome (Fig. 5). When DBF concentrations are zero (i.e. for TMT monotherapies) the activated ERK levels do not vary between 8 


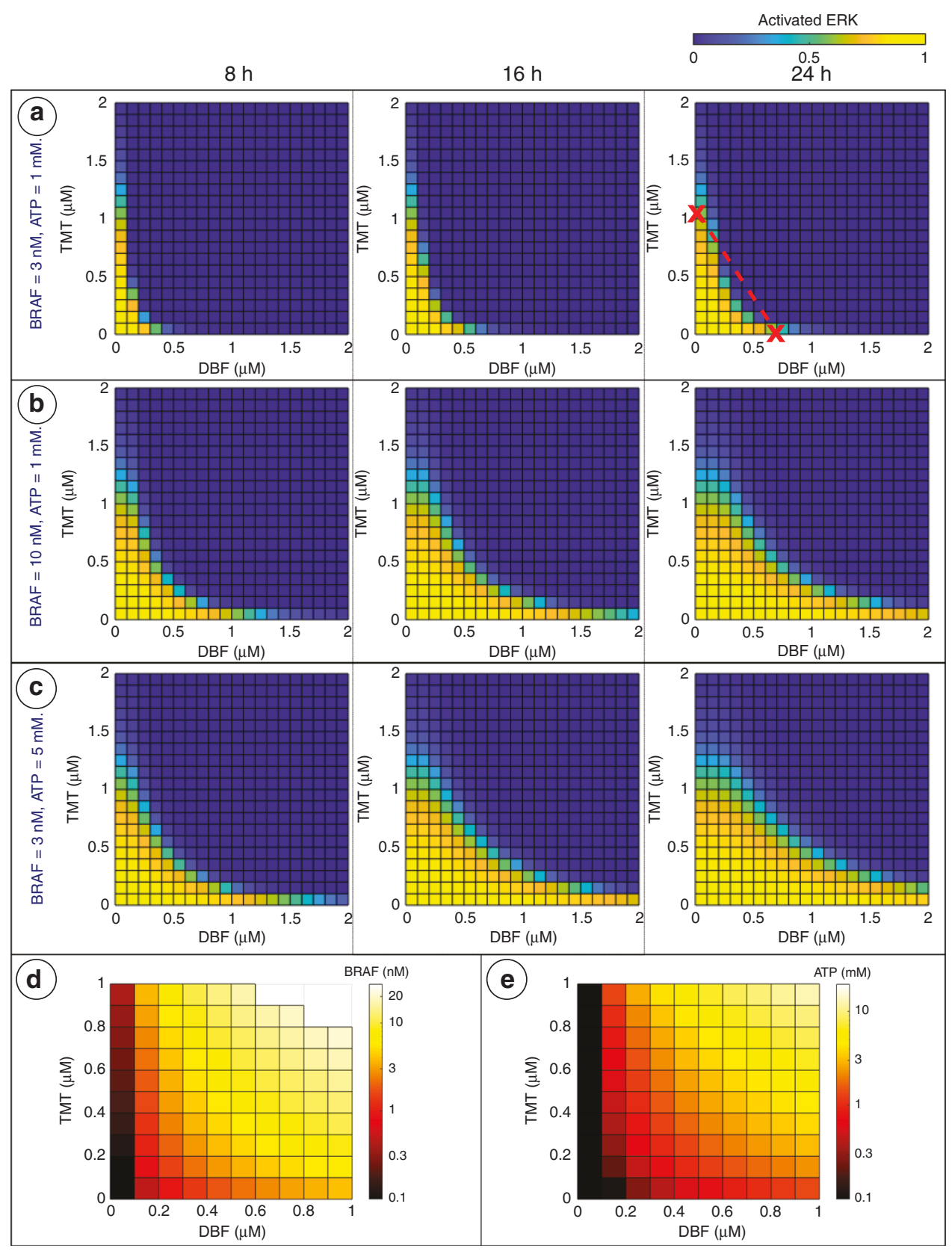

Fig. 5 Heatmaps showing treatment responses to dabrafenib-trametinib (DBF-TMT) combination therapies. For each square in the heatmap, the horizontal axis of the intersection denotes the dabrafenib dose, and the vertical axis denotes the trametinib dose. a-c The fraction of activated ERK (as defined in Eq. 2) at 8, 16 and $24 \mathrm{~h}$ are shown in the left, middle and right columns, respectively. BRAF and ATP concentrations vary between (a), (b) and (c). The right panel in (a) includes an isobole that connects the two monotherapy drug doses that yield an activated ERK level of 0.5 with a straight red line. Drug combinations below this line that yield activated ERK levels of 0.5 or less are categorised as synergistic. d, e The minimum BRAF (d) and ATP (e) concentrations that are required to yield an activated ERK level over 0.5 at $24 \mathrm{~h}$ are shown. In d, the ATP concentration is fixed at $1 \mathrm{mM}$. In (e), the BRAF concentration is fixed at $3 \mathrm{nM}$. For DBF-TMT combination doses where the activated ERK does not surpass 0.5 for BRAF concentrations under $20 \mathrm{nM}$, the squares in the heatmap in (d) are not coloured.

and $24 \mathrm{~h}$. However, when TMT concentrations are zero (i.e. for DBF monotherapies), the activated ERK levels do vary between 8 and $24 \mathrm{~h}$ and, as a consequence, so does DBF-TMT combination therapies (Fig. 5a-c).

In order to determine whether there is DBF-TMT synergism, we included an isobole that connects the two monotherapy doses that yield an activated ERK level of 0.5 (Fig. $5 \mathrm{a}$ ). Since drug combinations below this line can achieve activated ERK levels lower than 0.5 , the drug combination is categorised as synergistic [34].

In Fig. 5b, $c$, the BRAF and ATP levels have been increased from the baseline parameter values. By comparing Fig. 5 a with Fig. 5b, c, we can see that activated ERK levels increase with increased BRAF and ATP levels. Our mathematical model, therefore, predicts that increased intracellular BRAF and ATP concentrations will generate resistance to DBF-TMT combination therapies.

The influence of BRAF concentration on DBF-TMT efficacy is also reflected in Fig. $5 \mathrm{~d}$. We see from these results the minimum BRAF concentration that is required to achieve an activated ERK level of 0.5 at $24 \mathrm{~h}$ (when ATP is fixed at the baseline value $1 \mathrm{mM}$ ). Similarly, the minimum ATP concentration that is required to achieve an activated ERK level of 0.5 at $24 \mathrm{~h}$ (when BRAF is fixed at the baseline value $3 \mathrm{nM}$ ) is visualised in Fig. $5 \mathrm{e}$. 


\section{DISCUSSION}

Although targeted anticancer drugs can generate patient responses in tumours previously intractable to treatment, there remain major issues in their clinical use. In particular, tumour regrowth may occur due to a rapid onset of drug resistance. A number of mechanisms of drug resistance have been described for example for drugs targeted at mutant BRAF in the MAPK pathway. Many of these involve the modulation of components of the pathway, e.g. by mutation or gene amplification. This results in the sustained activation of ERK in the presence of drugs. As a consequence, drugs targeted at multiple components of the same pathway have been used in combination, e.g. BRAF and MEK inhibitors. Although this has resulted in some patient benefit, this has not resolved the problem and major challenges still remain. There is currently great interest in further developing the complexity of combination treatments. In the case of the BRAF pathway, e.g. by the addition of an inhibitor of ERK [4].

Increasing treatment complexity, however, raises some major pharmacological challenges, including which drugs to use in combination and what doses to use in order to keep toxicity at an acceptable level. At clinically administered doses, BRAF and MEK inhibitors lead to a number of side effects that are generally categorised to be non-life-threatening and safe when adequately monitored $[35,36]$. Common adverse events induced by DBF include hyperkeratosis, headache, arthralgia and pyrexia, whilst TMT commonly induces rash, diarrhoea, fatigue, peripheral oedema and acneiform dermatitis. DBF-TMT combination regimens have not been reported to yield any new adverse events. However, fever, chills, fatigue, diarrhoea, hypertension and vomiting are more frequently observed in response to combination therapies than in response to the corresponding monotherapies. On the contrary, cutaneous adverse events, such as squamous cell carcinoma and skin papilloma, occur less frequently in response to DBF-TMT combination therapies than in response to DBF monotherapies [35].

The vast number of drug combinations and dosing regimens that could be evaluated makes it impossible to test all these either in the laboratory or in the clinic. Informative in silico pharmacological models are therefore needed.

Several research groups have analysed and modified Huang and Ferrell's MAPK cascade model since it was presented in 1996 [21], as is summarised in a review by Orton et al. [2]. Mathematical analysis and computational simulation of the MAPK cascade model has explained how the dual phosphorylation and dephosphorylation events mechanistically give rise to the ultrasensitivity of ERK activation [37, 38] and bistability [39]. Integrated in vitromathematical work has further demonstrated switch-like (on or off) ERK activity in single cells in Xenopus oocytes subjected to progesterone [40]. Moreover, MAPK cascade models of various complexities have been presented and investigated in the literature, where these have included, e.g. the internalisation and downstream signalling activity of the epidermal growth factor receptor [41, 42].

To our knowledge, the mathematical model presented in this paper is the first MAPK cascade model to (i) specifically capture signalling dynamics in the BRAFV600E-MEK-ERK cascade, (ii) explicitly include ATP-dependent substrate phosphorylation, and (iii) include mechanistic drug actions of DBF and TMT, where the model parameters involved in (i-iii) are obtained from published in vitro data.

In this paper, we have developed a mathematical model that captures signalling dynamics in the BRAFV600E-MEK-ERK cascade, in response to DBF and TMT treatments. Our in silico model can be used to quantify the temporal evolution of system molecule concentrations and, as a consequence, ERK activity. The model predicts that increased cellular BRAF and ATP concentrations will result in reduced sensitivity to DBF-TMT combination therapies. The prediction that ATP levels may influence drug sensitivity

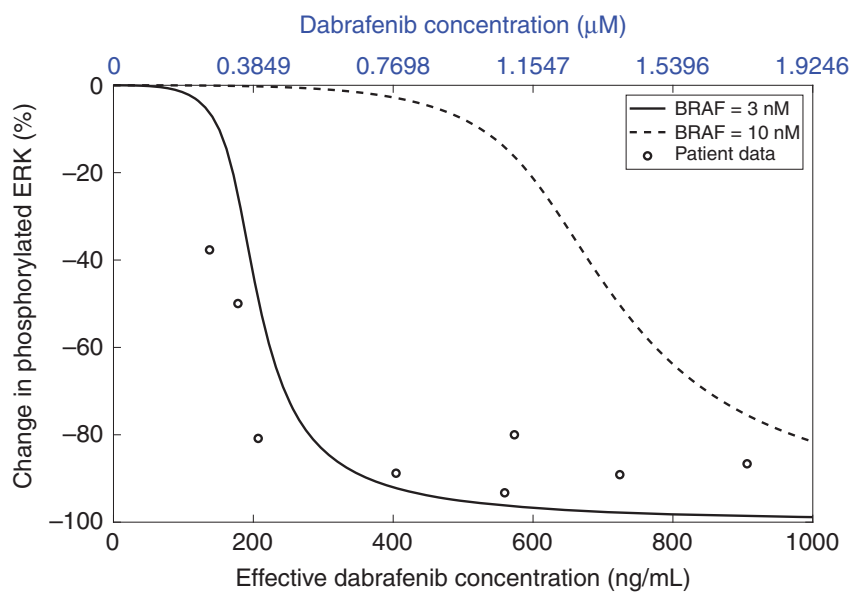

Fig. 6 Plots showing changes in phosphorylated ERK ([pERK] + [ppERK]) from the baseline (no drug) level. Simulation results at $8 \mathrm{~h}$, when the ATP concentration is fixed at $1 \mathrm{mM}$, are shown for BRAF concentrations of 3 and $10 \mathrm{nM}$. The circles show patient data from a clinical study by Falchook et al. [45], where dabrafenib was administered twice daily at doses between 70 and $200 \mathrm{mg}$. Dabrafenib concentrations are shown in $\mu \mathrm{M}$ (used in other simulations results in this article) along the top axis and $\mathrm{ng} / \mathrm{mL}$ (the effective concentration measured in the clinical study) along the bottom axis. To convert between $\mu \mathrm{M}$ and $\mathrm{ng} / \mathrm{mL}$, we have used that the molecular weight of dabrafenib is $519.6 \mathrm{~g} / \mathrm{mol}$ [53].

introduces an important variable to take into consideration when evaluating treatment responses. Intratumoural heterogeneity generated by both spatio-temporal factors (such as varying ATP levels within a tumour) and phenotypic factors (such as differences in BRAF amplification between cells) has been recognised to fuel drug resistance and complicate the design of treatment strategies $[43,44]$. Our finding that increased BRAF concentration is associated with drug resistance is furthermore consistent with experimental data [4].

Whilst simulation of the ERK activation level at different concentrations of DBF and $3 \mathrm{nM}$ BRAF closely followed that observed in patients [45], an increase of the BRAF level to $10 \mathrm{nM}$ in our model, similar to that found in drug-resistant patient-derived xenografts [4], predicted marked resistance of the ERK activation to treatment with DBF. This is shown in Fig. 6, where simulation results are superimposed over clinical data obtained from a study in which phosphorylated ERK levels were measured in BRAFV600positive melanoma biopsy samples before, and 5 days after, DBF monotherapy administration where doses between 70 and 200 $\mathrm{mg}$ were administered twice daily [45]. The recommended DBF dose for unresectable or metastatic BRAFV600E-positive melanoma is $150 \mathrm{mg}$ twice daily [6].

In order to investigate three-drug vertical inhibition of the BRAF-MEK-ERK pathway in BRAFV600E-mutant melanoma, the model could directly be extended to include the effects of an ERK inhibitor. As a further development of the modelling, one could study the effects of DBF-TMT treatment scheduling in a multicellular system using a multi-scale, agent-based model (ABM) $[46,47]$. In order to implement the pathway model into an $A B M$, the relationship between ERK activity and cell-cycle progression/ inhibition and cell death needs to be established [32, 33, 48]. This is one aim of our future modelling studies. ABMs are naturally able to incorporate spatio-temporal variations in intracellular BRAF and ATP concentrations amongst cells, where these variations are derived from both genetic, phenotypic and environmental factors $[49,50]$. ABMs can thus be used to simulate how drug-resistant tumour subclones (e.g. melanoma cells with elevated BRAF levels) and drug-sensitive tumour subclones evolve in time and space in response to various drug combinations, drug doses and drug 
treatment schedules. As an alternative future model extension to spatially explicit and stochastic ABMs, the model developed in the study can be integrated within a deterministic, age-structured cell population model in which the cell-cycle dynamics is dependent on ERK activity.

Thus, via a data-driven and bottom-up modelling approach [51], the in silico model developed in this study can be built upon to simulate how BRAF-MEK-ERK inhibition affects not only intracellular ERK activity but also the progression of drug resistance in melanoma tumours. Previous experimental work has shown that drug combinations, drug doses and treatment schedules all impact the evolution of drug-resistant tumour subclones in melanoma when the tumours are subjected to inhibitors that target the BRAF-MEK-ERK pathway $[4,52]$.

The predictive power of quantitative in silico models, such as that described here, is dependent on the accuracy of the kinetic constants for the different components of the signalling cascade as well as on the relative concentrations of the individual enzymes in the cascade. We would suggest that in the light of their key importance further studies are needed to validate the accuracy of the kinetic constants used. Indeed, translating such models to the treatment of individual cancer patients in the future may require the concentration and activity of the signalling components to be measured on an individual basis.

The mathematical model described in this study provides a method to quantitatively assess how vertical DFB-TMT inhibition suppresses ERK activity. We note that the modelling approach outlined in this paper can also be applied to other signalling cascades targeted in cancer treatment. Mathematical oncology models that are developed alongside experiments can be used to systematically motivate which drug combinations, drug doses and treatment schedules warrant experimental investigation [47].

\section{CODE AVAILABILITY}

Simulation code is available on the code hosting platform GitHub. Instructions on how to access, run and modify the code are available in the Supplementary Material (SM2)

\section{REFERENCES}

1. Cargnello M, Roux PP. Activation and function of the MAPKs and their substrates, the MAPK-activated protein kinases. Microbiol Mol Biol Rev. 2011;75:50-83.

2. Orton RJ, Sturm OE, Vyshemirsky V, Calder M, Gilbert DR, Kolch W. Computational modelling of the receptor-tyrosine-kinase-activated MAPK pathway. Biochem J. 2005:392:249-61.

3. Davies H, Bignell GR, Cox C, Stephens P, Edkins S, Clegg S, et al. Mutations of the BRAF gene in human cancer. Nature. 2002;417:949-54.

4. Xue $Y$, Martelotto $L$, Baslan $T$, Vides $A$, Solomon $M$, Mai $T$, et al. An approach to suppress the evolution of resistance in BRAFV600E-mutant cancer. Nat Med. 2017;23:929-37.

5. Karasarides M, Chiloeches A, Hayward R, Niculescu-Duvaz D, Scanlon I, Friedlos F, et al. B-RAF is a therapeutic target in melanoma. Oncogene. 2004;23:6292-8.

6. McGettigan S. Dabrafenib: a new therapy for use in BRAF-mutated metastatic melanoma. J Adv Pract Oncol. 2014;5:211-5.

7. Degirmenci U, Wang M, Hu J. Targeting Aberrant RAS/RAF/MEK/ERK Signaling for Cancer Therapy. Cells. 2020;9:198.

8. Ozkan-Dagliyan I, Diehl JN, George SD, Schaefer A, Papke B, Klotz-Noack K, et al. Low-dose vertical inhibition of the RAF-MEK-ERK cascade causes apoptotic death of KRAS mutant cancers. Cell Rep. 2020;31:107764.

9. Luke JJ, Hodi FS. Ipilimumab, vemurafenib, dabrafenib, and trametinib: synergistic competitors in the clinical management of BRAF mutant malignant melanoma. Oncologist. 2013;18:717-25.

10. Caunt CJ, Sale MJ, Smith PD, Cook SJ. MEK1 and MEK2 inhibitors and cancer therapy: the long and winding road. Nat Rev Cancer. 2015;15:577-92.

11. Shelledy L, Roman D. Vemurafenib: first-in-class BRAF-mutated inhibitor for the treatment of unresectable or metastatic melanoma. J Adv Pract Oncol. 2015;6:361-5

12. Tanda ET, Vanni I, Boutros A, Andreotti V, Bruno W, Ghiorzo P, et al. Current state of target treatment in BRAF mutated melanoma. Front Mol Biosci. 2020;7:154.
13. Robert C, Karaszewska B, Schachter J, Rutkowski P, Mackiewicz A, Stroiakovski D, et al. Improved overall survival in melanoma with combined dabrafenib and trametinib. N Engl J Med. 2015;372:30-9.

14. Long GV, Stroyakovskiy D, Gogas H, Levchenko E, de Braud F, Larkin J, et al. Combined BRAF and MEK inhibition versus BRAF inhibition alone in melanoma. $\mathrm{N}$ Engl J Med. 2014;371:1877-88.

15. Rizos H, Menzies AM, Pupo GM, Carlino MS, Fung C, Hyman J, et al. BRAF inhibitor resistance mechanisms in metastatic melanoma: spectrum and clinical impact. Clin Cancer Res. 2014;20:1965-77.

16. Queirolo P, Spagnolo F. BRAF plus MEK-targeted drugs: a new standard of treatment for BRAF-mutant advanced melanoma. Cancer Metastasis Rev. 2017;36:35-42.

17. Ascierto PA, Dummer R, Gogas HJ, Flaherty KT, Arance A, Mandala M, et al. Update on tolerability and overall survival in COLUMBUS: landmark analysis of a randomised phase 3 trial of encorafenib plus binimetinib vs vemurafenib or encorafenib in patients with BRAF V600-mutant melanoma. Eur J Cancer. 2020;126:33-44.

18. Luebker SA, Koepsell SA. Diverse mechanisms of BRAF inhibitor resistance in melanoma identified in clinical and preclinical studies. Front Oncol. 2019;9:1-8.

19. Johnson DB, Menzies AM, Zimmer L, Eroglu Z, Ye F, Zhao S, et al. Acquired BRAF inhibitor resistance: a multicenter meta-analysis of the spectrum and frequencies, clinical behaviour, and phenotypic associations of resistance mechanisms. Eur J Cancer. 2015;51:2792-9.

20. Sale MJ, Balmanno K, Saxena J, Ozono E, Wojdyla K, McIntyre RE, et al. MEK1/2 inhibitor withdrawal reverses acquired resistance driven by BRAFV600E amplification whereas KRASG13D amplification promotes EMT-chemoresistance. Nat Commun. 2019;10:2030.

21. Huang $C Y$, Ferrell JE. Ultrasensitivity in the mitogen-activated protein kinase cascade. Proc Natl Acad Sci USA. 1996;93:10078-83.

22. VanScyoc WS, Holdgate GA, Sullivan JE, Ward WH. Enzyme kinetics and binding studies on inhibitors of MEK protein kinase. Biochemistry. 2008;47:5017-27.

23. Hatzivassiliou G, Song K, Yen I, Brandhuber BJ, Anderson DJ, Alvarado R, et al. RAF inhibitors prime wild-type RAF to activate the MAPK pathway and enhance growth. Nature. 2010;464:431-5.

24. Food FDA (US, Administration) D. Reference ID: 4255750 [Internet]. 2018. https// www.accessdata.fda.gov/drugsatfda/_docs/label/2018/202806s008lbl.pdf. Accessed 14 Feb 2020.

25. Rheault TR, Stellwagen JC, Adjabeng GM, Hornberger KR, Petrov KG, Waterson $A G$, et al. Discovery of dabrafenib: a selective inhibitor of raf kinases with antitumor activity against B-Raf-driven tumors. ACS Med Chem Lett. 2013:4:358-62.

26. Tsai J, Lee JT, Wang W, Zhang J, Cho H, Mamo S, et al. Discovery of a selective inhibitor of oncogenic B-Raf kinase with potent antimelanoma activity. Proc Natl Acad Sci USA. 2008;105:3041-6.

27. Koelblinger $P$, Thuerigen $O$, Dummer R. Development of encorafenib for BRAFmutated advanced melanoma. Curr Opin Oncol. 2018;30:125-33.

28. Mansour SJ, Candia JM, Matsuura JE, Manning MC, Ahn NG. Interdependent domains controlling the enzymatic activity of mitogen-activated protein kinase kinase 1. Biochemistry. 1996;35:15529-36.

29. Gilmartin AG, Bleam MR, Groy A, Moss KG, Minthorn EA, Kulkarni SG, et al GSK1120212 (JTP-74057) is an inhibitor of MEK activity and activation with favorable pharmacokinetic properties for sustained in vivo pathway inhibition. Clin Cancer Res. 2011;17:989-1000.

30. King AJ, Arnone MR, Bleam MR, Moss KG, Yang J, Fedorowicz KE, et al. Dabrafenib; preclinical characterization, increased efficacy when combined with trametinib, while BRAF/MEK tool combination reduced skin lesions. PLoS ONE. 2013;8: e67583.

31. Roskoski R. Allosteric MEK $1 / 2$ inhibitors including cobimetanib and trametinib in the treatment of cutaneous melanomas. Pharmacol Res. 2017;117:20-31.

32. Yamamoto T, Ebisuya M, Ashida F, Okamoto K, Yonehara S, Nishida E. Continuous ERK activation downregulates antiproliferative genes throughout $\mathrm{G} 1$ phase to allow cell-cycle progression. Curr Biol. 2006;16:1171-82.

33. Chambard JC, Lefloch R, Pouysségur J, Lenormand P. ERK implication in cell cycle regulation. Biochim Biophys Acta. 2007;1773:1299-310.

34. Tallarida RJ. Quantitative methods for assessing drug synergism. Genes Cancer 2011;2:1003-8.

35. Welsh SJ, Corrie PG. Management of BRAF and MEK inhibitor toxicities in patients with metastatic melanoma. Ther Adv Med Oncol. 2015;7:122-36.

36. Heinzerling L, Eigentler TK, Fluck M, Hassel JC, Heller-Schenck D, Leipe J, et al. Tolerability of BRAF/MEK inhibitor combinations: adverse event evaluation and management. ESMO Open. 2019;4:e000491.

37. Burack WR, Sturgill TW. The activating dual phosphorylation of MAPK by MEK is nonprocessive. Biochemistry. 1997;36:5929-33.

38. Ferrell JE, Bhatt RR. Mechanistic studies of the dual phosphorylation of mitogenactivated protein kinase. J Biol Chem. 1997;272:19008-16. 
39. Markevich NI, Hoek JB, Kholodenko BN. Signaling switches and bistability arising from multisite phosphorylation in protein kinase cascades. J Cell Biol. 2004;164:353-9.

40. Ferrell JE, Machleder EM. The biochemical basis of an all-or-none cell fate switch in Xenopus oocytes. Science. 1998;280:895-8.

41. Brightman FA, Fell DA. Differential feedback regulation of the MAPK cascade underlies the quantitative differences in EGF and NGF signalling in PC12 cells. FEBS Lett. 2000;482:169-74.

42. Schoeberl B, Eichler-Jonsson C, Gilles ED, Müller G. Computational modeling of the dynamics of the MAP kinase cascade activated by surface and internalized EGF receptors. Nat Biotechnol. 2002;20:370-5.

43. Roesch A. Tumor heterogeneity and plasticity as elusive drivers for resistance to MAPK pathway inhibition in melanoma. Oncogene. 2015;4:2951-7.

44. Dagogo-Jack I, Shaw AT. Tumour heterogeneity and resistance to cancer therapies. Nat Rev Clin Oncol. 2017;15:81-94.

45. Falchook GS, Long GV, Kurzrock R, Kim KB, Arkenau HT, Brown MP, et al. Dose selection, pharmacokinetics, and pharmacodynamics of BRAF inhibitor dabrafenib (GSK2118436). Clin Cancer Res. 2014;20:4449-58.

46. Rejniak KA, Anderson ARA. Hybrid models of tumor growth. Wiley Interdiscip Rev Syst Biol Med. 2011;3:115-25.

47. Rockne RC, Hawkins-Daarud A, Swanson KR, Sluka JP, Glazier JA, Macklin P, et al. The 2019 mathematical oncology roadmap. Phys Biol. 2019;16:41005.

48. Mebratu Y, Tesfaigzi Y. How ERK1/2 activation controls cell proliferation and cell death: Is subcellular localization the answer? Cell Cycle. 2009;8:1168-75.

49. Hamis S, Nithiarasu P, Powathil GG. What does not kill a tumour may make it stronger: In silico insights into chemotherapeutic drug resistance. J Theor Biol. 2018;454:253-67.

50. Hamis S, Kohandel M, Dubois LJ, Yaromina A, Lambin P, Powathil GG. Combining hypoxia-activated prodrugs and radiotherapy in silico: Impact of treatment scheduling and the intra-tumoural oxygen landscape. PLoS Comput Biol. 2020;16: e1008041.

51. Hamis S, Powathil GG, Chaplain MAJ. Blackboard to bedside: a mathematical modeling bottom-up approach toward personalized cancer treatments. JCO Clin Cancer Inf. 2019;3:1-11.

52. Smalley I, Kim E, Li J, Spence P, Wyatt CJ, Eroglu Z, et al. Leveraging transcriptional dynamics to improve BRAF inhibitor responses in melanoma. EBioMedicine. 2019;48:178-90.

53. National Center for Biotechnology Information. PubChem Compound Summary for CID 44462760, Dabrafenib. [Internet]. 2021. https://pubchem.ncbi.nlm.nih. gov/compound/Dabrafenib. Accessed 11 Jul 2021.

\section{ACKNOWLEDGEMENTS}

A prior preprint version of this article is available on the preprint server bioRxiv (https://doi.org/10.1101/2021.04.20.440559).

\section{AUTHOR CONTRIBUTIONS}

All authors designed the study and contributed to the mathematical model. SJH carried out the computational simulations. All authors analysed the data and results.
SJH drafted the manuscript. All authors revised the manuscript. All authors have approved the manuscript, and have agreed to be accountable for all aspects of the work presented in the manuscript.

\section{FUNDING INFORMATION}

All authors acknowledge support from the Medical Research Council [grant code MR/ R017506/1].

\section{ETHICS APPROVAL AND CONSENT TO PARTICIPATE}

Not applicable.

\section{CONSENT TO PUBLISH}

Not applicable.

\section{COMPETING INTERESTS}

The authors declare no competing interests.

\section{ADDITIONAL INFORMATION}

Supplementary information The online version contains supplementary material available at https://doi.org/10.1038/s41416-021-01565-w.

Correspondence and requests for materials should be addressed to Sara J. Hamis.

Reprints and permission information is available at http://www.nature.com/ reprints

Publisher's note Springer Nature remains neutral with regard to jurisdictional claims in published maps and institutional affiliations.

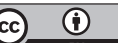

Open Access This article is licensed under a Creative Commons Attribution 4.0 International License, which permits use, sharing, adaptation, distribution and reproduction in any medium or format, as long as you give appropriate credit to the original author(s) and the source, provide a link to the Creative Commons license, and indicate if changes were made. The images or other third party material in this article are included in the article's Creative Commons license, unless indicated otherwise in a credit line to the material. If material is not included in the article's Creative Commons license and your intended use is not permitted by statutory regulation or exceeds the permitted use, you will need to obtain permission directly from the copyright holder. To view a copy of this license, visit http://creativecommons. org/licenses/by/4.0/.

(c) The Author(s) 2021 